\title{
AlCALOIDES EN LA FLORA DE ANDALUCIA. III
}

\author{
Rafael SUAU, Ana I. GARCIA, Rodrigo RICO, Baltasar CABEZUDO, \\ José M. NIETO-CALDERA \& A. Enrique SALVO
}

\begin{abstract}
RESUMEN: Se han estudiado 79 especies vegetales características de la flora de Andalucía con objeto de determinar la presencia cualitativa y cuantitativa de alcaloides. Se ha encontrado presencia de alcaloides en 16 especies, de las cuales 6 se describen por primera vez. Se han registrado los espectros de masas de los extractos alcaloídicos de varias especies y se ha establecido su relación con los alcaloides presentes.
\end{abstract}

Palabras clave: Alcaloides, espectrometría de masas, flora de Andalucía, España.

SUMMARY: 79 different species from the Andalusian flora have been screened for the presence of alkaloids. Of these, 16 species were found to contain alkaloids and 6 of them are reported for the first time. A relationship between mass spectra and alkaloid contents has been established for several species.

Key words: Alkaloids, mass spectrometry, Andalusian flora, Spain.

\section{N T R O D U C C I O N}

Continuamos en este trabajo nuestras investigaciones sobre la presencia de alcaloides en plantas vasculares de la Flora de Andalucía (1,2). Junto a la determinación cualitativa puesta de manifiesto por acción de reactivos precipitantes y su valoración cuantitativa, en este tercer trabajo hemos llevado a cabo el estudio de algunas fracciones de alcaloides por espectrometría de masas, con introducción directa de muestras y con ionización por impacto electrónico. Los resultados proporcionan una información adicional que en algunos casos permite predecir el tipo de alcaloides contenidos en la especie vegetal. Este diagnóstico permite a su vez discutir la bondad de algunas determinaciones taxonómicas anteriores así como el grado de relación entre especies próximas.

\section{MET ODOLOG I A}

El procedimiento experimental seguido en el secado del material vegetal, su extracción, aislamiento de la fracción de alcaloides, ensayos cualitativos y valoración 
cuantitativa ha sido el mismo que el descrito anteriormente $(1,2)$.

Para aquellas muestras en las que la cantidad de material disponible ha sido menor de $5 \mathrm{~g}$ de planta seca, se ha empleado un extractor soxhlet de $50 \mathrm{ml}$ de capacidad, reduciendo los volúmenes utilizados en el proceso a una quinta parte.

La reacción de precipitación se considera $[+++]$ cuando al adicionar los reactivos se forma un precipitado abundante con o sin floculación y se considera [++] cuando se forma una turbidez claramente definida y sin floculación. En ambos casos la valoración con ácido p-toluen-sulfónico es superior a 0.4 miliequivalentes (mEq) de alcaloides por $100 \mathrm{~g}$ de planta seca, y una conclusión positiva (P) sobre el contenido en alcaloides. La reacción es [+] cuando se forma una ligera, pero persistente, opacidad. La valoración define una conclusión positiva si es superior a $0.2 \mathrm{mEq}$, si es inferior a este valor, se considera una conclusión dudosa (D). Un ligero enturbiamiento $[(+)]$ o su ausencia establecen una conclusión negativa $(\mathrm{N})$.

Los espectros de masas han sido registrados en un espectrómetro HewlettPackard 5988 A. Las fracciones de alcaloides disueltas en cloroformo/metanol (1:1), se aplican a una sonda de introducción directa, programada con una velocidad de calentamiento de $50^{\circ} \mathrm{C} /$ minuto, hasta $250^{\circ} \mathrm{C}$. Las muestras fueron ionizadas a $70 \mathrm{eV}$ y se registraron los iones totales en un rango de masas comprendido entre $\mathrm{m} / \mathrm{z} 50 \mathrm{y}$ $\mathrm{m} / \mathrm{z} 550$. Los resultados expresados en la tabla II se refieren a la suma de todos los barridos registrados para cada muestra (entre 200 y 300 ).

En la zona de masas altas los iones de $\mathrm{m} / \mathrm{z}$ impar pueden ser asociados a iones moleculares $(\mathrm{m} / \mathrm{z}=$ peso molecular, para $\mathrm{z}=1)$ correspondientes a alcaloides con un único átomo de nitrógeno (o menos frecuente un número impar de átomos de nitrógeno). En esta misma zona, la presència de iones significativos de $\mathrm{m} / \mathrm{z}$ par puede ser interpretado como iones moleculares correspondientes a alcaloides con dos átomos de nitrógeno (o un número par de átomos de nitrógeno) o con un nitrógeno en forma de sal cuaternaria. En ningún caso puede descartarse que la señal observada corresponda a un proceso de fragmentación.

En la zona de masas medias y bajas del espectro se observan los iones correspondientes a las fragmentaciones más características y que pueden expresar información básica sobre los tipos de alcaloides o confirmación de los mismos (3).

\section{A T E R I A L E S T U D I A D O}

Al final del trabajo (addenda I) se relacionan por orden alfabético, todas las especies estudiadas y se establece para cada una de ellas, lugar y fecha de recolección así como el número de registro de entrada en el herbario del Dpto. de Biología Vegetal de la Universidad de Málaga (MGC), donde todo el material se encuentra depositado como testigo.

La procedencia de parte del material estudiado en la Tabla II ha sido reseñada en trabajos anteriores $(1,2)$, el resto corresponde al mismo material de la Tabla I (addenda I).

\section{R E S UL T A D O S}

En la Tabla I se describen los resultados obtenidos en la valoración. de las distintas especies estudiadas, expresándose los resultados de la valoración cuantitativa en 
$\mathrm{mEq}$ de alcaloides por cada $100 \mathrm{~g}$ de planta seca.

En la Tabla II se describen los espectros de masas de los extractos de alcaloides de algunas especies que han mostrado una valoración positiva, ya sea en éste o en trabajos anteriores $(1,2)$. Las especies se relacionan alfabéticamente y se da la referencia bibliográfica para la localización del material vegetal empleado.

De los espectros de masas se han tomado las señales $(\mathrm{m} / \mathrm{z}$, para $\mathrm{z}=1)$ más significativas, con indicación de sus abundancias relativas referidas en todos los casos al pico base del espectro ( $100 \%$ de abundancia relativa).

\section{O N C L US I O N E S}

Tabla I.- De las 79 muestras estudiadas correspondientes a 75 especies, 16 (20\%) dieron resultado positivo, 10 (13\%) dieron resultado dudoso y $53(67 \%)$ arrojaron resultado negativo. No se han encontrado trabajos previos para el $71 \%$ de las especies que se describen.

Se confirma en algunos casos una amplia variabilidad en los contenidos de alcaloides al comparar distintas poblaciones de una misma especie. Así, el resultado positivo obtenido con una nueva población de Santolina chamaecyparissus (Asteraceae) contrasta con el resultado negativo obtenido en otra población estudiada anteriormente (1) y parece confirmar la presencia de alcaloides en el género Santolina.

Por otra parte, una nueva población de Moricandia moricandioides (Brassicaceae) ha resultado claramente negativa, mientras que otra población de $M o$ ricandia arvensis ha resultado dudosa, ambos casos presentan un manifiesto contraste con resultados anteriores (1) y requerirán de posteriores estudios.

Llama la atención la ausencia de alcaloides en las dos poblaciones estudiadas de Maytenus senegalensis (Celastraceae), a pesar de que en esta especie, y en el género Maytenus de forma general se encuentran maytansinoides, alcaloides macrocíclicos de importante actividad antitumoral (7). Es posible que su baja solubilidad en medio ácido y su baja sensibilidad frente a los reactivos precipitantes explique este resultado.

Es de notar la gran variabilidad, en cuanto al contenido en alcaloides, que se produce en la familia Scrophulariaceae. En el género Scrophularia, junto a especies con una elevada concentración de alcaloides ( . lyrata) se dan otras especies con contenido muy pequeño o incluso ausencia total ( $S$. crithmifolia). Análoga situación muestra el género Chaenorrhinum, junto a $C$. villosum que es claramente positivo (1), el $C$. glareosum es negativo, mientras que para una misma población de $C$. macropodium subsp. degenii, las partes aéreas dan positivo y la planta total da negativo, probablemente debido a un efecto de dilución del material vegetal, junto a la ausencia de alcaloides en las raíces.

Tabla II.- En Platicapnos tenuiloba subsp. tenuiloba (Papaveraceae) se observan los posibles iones moleculares 355 y 339, y los fragmentos (M-1) a 354 y 338 , que sugieren alcaloides con esqueleto aporfínico (3), con metoxilos como sustituyentes, según puede desprenderse de la presencia de iones intensos a M-15 (340 y 324). En este caso el resultado se ha confirmado por aislamiento de los alcaloides 
(17).

Otra Papaveraceae, Sarcocapnos baetica subsp. baetica muestra un espectro totalmente distinto. En él se puede interpretar el grupo de iones moleculares a 355 y 353 asociado a un pico base $\mathrm{m} / \mathrm{z} 148$, con la presencia de alcaloides protopínicos, mientras que el ion molecular 327 y su fragmento 312 se pueden asociar a la presencia de alcaloides cularínicos, los cuales parecen estar estrechamente relacionados con el género Sarcocapnos (18).

El género Senecio (Asteraceae) contiene un grupo muy homogéneo de alcaloides con esqueleto pirrolizidínico (19). El espectro de masas del extracto de alcaloides de $S$. pyrenaicus subsp. granatensis presenta dos iones moleculares aparentes, 353 y 337. La diferencia en 16 unidades de masas entre ellos sugiere la estructura de un N-óxido para el primero de ellos. Resultado que se ha confirmado recientemente (17).

Los iones abundantes de masa par en Hedysarum coronarium sugieren la presencia de alcaloides quinolizidínicos con dos átomos de nitrógeno, frecuentes en otros géneros de Fabaceae como Lupinus, Genista o Retama (20).

En otros casos la interpretación global de los espectros es más compleja, especialmente cuando presenta iones moleculares más imprecisos y poco significativos debido a fragmentaciones fáciles. Tal es la situación en los alcaloides diterpénicos presentes en Delphinium (Ranunculaceae) o en los de Narcissus (Amaryllidaceae) $(21,5)$. En estos últimos casos incluso es difícil encontrar analogías en los espectros de distintas especies del mismo género.

Un comportamiento análogo lo muestran las dos especies del género Chaenorrhinum (Scrophulariaceae). Todos los datos apuntan hacia alcaloides de elevado peso molecular, y varios átomos de nitrógeno, asegurando fáciles roturas que conducen a señales de difícil significación (22).

Los datos incluidos en la tabla II describen una propiedad intrínseca de cada especie y pueden ser empleados como ayuda taxonómica. En este sentido, hemos comprobado que una población de Papaver identificada inicialmente como Papaver dubium (17), muestra en su espectro de masas la ausencia total de los iones 279 y 278 que serían de esperar para roemerina, el alcaloide más abundante en esta especie (23). Los iones abundantes a 177 y 163 unidades de masa, parecen indicar la presencia de alcaloides con esqueleto de papaverrubinas y roeadinas (24), característicos de otras especies del género Papaver ( $P$. argemone, $P$. rhoeas, $P$. hibridum). La revisión morfológica del material vegetal ha confirmado que se trata de Papaver rhoeas y no de Papaver dubium.

La aplicación de estas metodologías en la investigación de alcaloides dentro de familias botánicas específicas, se encuentra en vias de realización.

\section{A D D E N D A I}

Abies pinsapo Boiss. Málaga: Tolox; 25.IV.86 (MGC 20725)

Anthyllis cytisoides L. Almería: Velez-Rubio; 27.IV.86 (MGC 23007)

Anthyllis gerardii L. Málaga: Marbella; 06.IV.88 (MGC 21587)

Anthyllis podocephala Boiss. Málaga: Marbella; 06.IV.86 (MGC 20721)

Antirrhinum australe Rothm. Málaga: Alpandeire; 27.III.88 (MGC 21551) 
Antirrhinum barrelieri Boreau. Málaga: Nerja; 17.V.86 (MGC 22991)

Arenaria delaguardiae G. López \& Nieto. Málaga: Cómpeta; 26. VI.86 (MGC 22995)

Astragalus lusitanicus Lam. Sevilla: Valdeflores; 04.IV.86 (MGC 20730)

Bupleurum fruticescens L. Almería: Velez-Rubio; 27.IV.86 (MGC 22988)

Calamintha sylvatica subsp. ascendens (Jordan) P.W. Ball. Cádiz: Cortes de la Frontera; 09.XI.86 (MGC 18206)

Chaenorrhinum glareosum (Boiss.) Willk. Granada: Sierra Nevada; 22.VII.87 (MGC 20645)

Chaenorrhinum macropodum (Boiss. \& Reuter) subsp. degenii (Hervier) R. Fernández (a). Málaga: Sierra Tejeda; 26.VI.86 (MGC 23006)

Chaenorrhinum macropodum (Boiss. \& Reuter) subsp. degenii (Hervier) R. Fernández (b). Málaga: Sierra Tejeda; 26.VI.86 (MGC 23005)

Cleonia lusitanica L. Málaga: Alhaurín de la Torre; 02.VI.87 (MGC 20698)

Coris monspeliensis L. Málaga: Cómpeta; 26.VI.86 (MGC 20687)

Coronilla juncea L. Málaga: Alpandeire; 27.III.88 (MGC 21554)

Daphne gnidium L. Málaga: Entre Almogía y Antequera; 24.X.86 (MGC 21540)

Daphne laureola L. subsp. latifolia (Cosson) R. Martínez. Málaga; Tolox; 24.IV.88 (MGC 23025)

Delphinium pentagynum Lam (a). Cádiz: Alcalá de los Gazules; 20.VI.88 (MGC 21612)

Delphinium pentagynum Lam (b). Málaga: Tolox; 07.VI;86 (MGC 21531)

Dianthus broteri Boiss. \& Reuter. Almería: Huercal-Overa; 04.VI.86 (MGC 21374)

Digitalis obscura var. laciniata Lindley. Málaga: Istán; 25.IV.86 (MGC 18218)

Digitalis obscura L. subsp. obscura. Granada: Sierra Tejeda; 27.III.87 (MGC 21604)

Ephedra gragilis Desf. Málaga: Alhaurín de la Torre; 22.II.86 (MGC 20661)

Ferula communis L. Granada: Motril; 18.IV.86 (MGC 18214)

Genista linifolia L. Málaga: Algatocín; 27.III.88 (MGC 21550)

Genista spartioides Spach. Málaga: Nerja; 17.V.86 (MGC 23001)

Hedysarum coronarium L. Málaga: Manilva; 28.III.88 (MGC 21560)

Hedysarum humile L. Málaga: Antequera; 19.V.86 (MGC 21530)

Helleborus foetidus L. Málaga: Tolox; 24.IV.88 (MGC 23022)

Hippocrepis rupestris Laza. Málaga: Sierra Tejeda; 27.III.87 (MGC 21593)

Hippocrepis scabra DC. Málaga: Antequera; 19.V.86 (MGC 21373)

Inula crithmizdes L. Cádiz: Puerto de Santa María; 20VI.88 (MGC 21616)

Launea resedifolia (L.) O. Kuntze. Almería: Huercal-Overa; 04.VI.86 (MGC 21628)

Lavandula stoechas L. subsp. sampaiana Rozeira. Sevilla: Valdeflores; 04.IV.86 (MGC 20729)

Lavatera maritima Gouan. Granada: Motril; 28.IV.86 (MGC 18213)

Limoniastrum monopetalum (L.) Boiss. Cádiz: Puerto de Santa María; 20.VI.88 (MGC 21614)

Limonium eugeniae Senen. Almería: Tabernas; 04.VI.86 (MGC 21629)

Linaria hirta (L.) Moench. Málaga: Antequera; 09.V.88 (MGC 21606)

Linaria latifolia Desf. Málaga: Antequera; 09.V.88 (MGC 21607)

Linaria satureioides Boiss. Málaga; Coín; 01.III.88 (MGC 21564)

Linaria spartea (L.) Chaz. Huelva: Almonte; 05.IV.86 (MGC. 21363)

Loeflingia baetica Lag. var. baetica: Huelva: Mazagón; 06.IV.86 (MGC 22996)

Lupinus micranthus Guss. Málaga: Algatocín; 27.III.88 (MGC 21549)

Lythrum salicaria L. Huelva: Almonte; 9.IX.87 (MGC 18249)

Maytenus senegalensis (Lam.) Exell (a). Granada: Rubite; 11.VI.88 (MGC 21621)

Maytenus senegalensis (Lam.) Exell (b). Granada: Almuñecar; 28.II.86 (MGC 20705)

Moricandia arvensis (L.) DC. Almería: Sorbas; 04.VI.86 (MGC 21528)

Moricandia moricandioides (Boiss.) Heywood. Málaga: El Burgo; 27.III.88 (MGC 21557)

Narcissus papyraceus Ker. Gawler subsp. polyanthus (Loisel.) Ascherson. Málaga: Alora; 17.I.87 (MGC 22992)

Nigella papillosa G. López subsp. papillosa. Málaga: Casabermeja; 10.VI.88 (MGC 21618) 
Orchis mascula (L.) subsp. olbiensis (Reuter ex Gren.) Ascherson \& Graebner. Málaga: Alora; 22.III.87 (MGC )

Orchis saccata Ten. Málaga: Alhaurín el Grande; 16.III.86 (MGC 20710)

Papaver rhoeas L. Málaga: Casabermeja; 10.VI.88 (MGC 21612)

Parentucellia viscosa (L.) Carvel. Málaga: Casares; 05.V.88 (MGC 21622)

Polygala rupestris Pourret. Málaga: Alora; 21.VI.86 (MGC 20694)

Potentilla petrophila Boiss. Granada: Sierra Nevada; 23.VII.87 (MGC 18265)

Prasium majus L. Granada: Motril; 18.IV.86 (MGC 18217)

Putoria calabrica (L. fil.) DC. Málaga: Yunquera; 07.VI.86 (MGC 20667)

Quercus faginea Lam. Málaga: Tolox; 25.IV.86 (MGC 20719)

Ranunculus spicatus Derf. subsp. blepharicarpos (Boiss.) Grau. Granada: Motril; 18.IV.86 (MGC 18212)

Rhamnus alaternus L. Málaga: entre El Chorro y Bobastro; 21.VI.86 (MGC 21537)

Rubia peregrina L. Málaga: Istán; 25.IV.86 (MGC 23030)

Rumex tingitanus L. Huelva: Mazagón; 06.IV.86 (MGC 21360)

Salvia barrelieri Etlinger. Cádiz: Alcalá de los Gazules; 20.VI.88 (MGC 21613)

Salvia verhenaca L. Granada: Guadix; 26.IV.86 (MGC 21349)

Santolina chamaecyparissus L. Granada: Sierra Tejeda; 27.III.87 (MGC 21600)

Sarcocapnos baetica (Boiss. \& Reuter) Nyman subsp. baetica. Málaga: El Burgo; 28.IV.88 (MGC 23047)

Satureja salzmannii P.W. Ball. Cádiz: Alcalá de los Gazules; 20.VI.88 (MGC 21619)

Saxifraga globulifera Desf. Málaga: Alhaurín el Grande; 16.III.86 (MGC 20716)

Scruphularia crithmifolia Boiss. Málaga: Alozaina; 27.III.88 (MGC 21556)

Scrophularia lyrata Willd. Huelva: Almonte; 9.IX.87 (MGC 18247)

Scrophularia sambucifolia L. Málaga: La Sauceda; 28.III.88 (MGC 21546)

Sideritis arborescens Salzm. subsp. arborescens. Málaga: Marbella; 06.IV.88 (MGC 21586)

Sideritis incana L. subsp. virgata (Desf.) Malarriga (a). Málaga: Sierra Almijara; 17,V.86 (MGC 23000)

Sideritis incana L. subsp. virgata (Desf.) Malagarriga (b). Granada: Sierra Nevada; 23.VII.87 (MGC 18266)

Silene colorata Poiret. Sevilla: Cazalla de la Sierra; 04.IV.86 (MGC 20728)

Thymus zygis subsp. gracilis (Boiss.) R. Morales. Málaga: Nerja; 17.V.86 (MGC 22989)

Vinca difformis Pourret. Málaga: Alpandeire; 17.III.88 (MGC 21553).

\section{B I B L I O G R A F I A}

1. SUAU, R., GARCIA, A.I., RICO, R., CABEZUDO, B., NIETO, J.M. \& SALVO, A.E. 1988- Alcaloides en la Flora de Andalucía. I. Acta Bot. Malacitana, 13:189.

2. SUAU, R., GARCIA, A.I., RICO, R., CABEZUDO, B., NIETO, J.M. \& SALVO, A.E. 1989- Alcaloides en la Flora de Andalucía, II. Acta Bot. Malacitana, 14:141.

3. BUDZIKIEWICZ, M., DJERASSI, C. \& WILLIAMS, D.H. -1964- Structure elucidation of natural products by Mass Spectrometry. Vol. I. Holden-Day. San Francisco.

4. SMOLENSKY, S.J., SILINIS, H. \& FARNSWORTH, N.R. -1975- Alkaloid screening. VI. Lloydia, 38:225.

5. SUAU, R., GARCIA, A.I., RICO, R. \& GOMEZ, A.I.-1990- New Amaryllidaceae alkaloids from Narcissus papyraceus Ker-Gawler. Heterocycles, 31:517.

6. GARNIER, J. \& MAHUTEAU, J. -1985- Difforlemenine, alcaloide nouveau de Vinca difformis. Determination de estructure par RMN a $400 \mathrm{MHz}$. Tet. Letters, 26:1513. 
7. TIN-WA, M., FARNSWORTH, N.R., FONG, H.H.S., BLOMSTER, R.N., TROJANEK. J., ABRAHAM, D.I., PERSINOS, G.J. \& DOKOSI, O.B. -1971- Biological and phytochemical evaluation of plants. IX. Antitumor activity of Maytenus senegalensis and a preliminary phytochemical investigation. Lloydia, 34:79.

8. SERRANO, M., CODINA, C., VILADOMAT, F., BASTIDA, J. \& LLABRES, J.M. 1985- Alkaloid screening of Catalonia (Spain) plants. II. Int. J. Crude Drug Res., 23:105.

9. VILADOMAT, F., CODINA, C., BASTIDA, J., GALOBARDES, M. \& SERRANO, M. -1984- Alkaloid screening of Catalonia (Spain) plants, I. Lloydia, 47:64.

10. VILADOMAT, F., CODINA, C., LLABRES, J.M. \& BASTIDA, J. -1986- Alkaloid screening of plants of Catalonia (Spain). III. Int. J. Crude Drug Res., 24:123.

11. WILLAMAN, J.J. \& LI, H.L. -1970- Alkaloids bearing plants and their contained alkaloids. 1957-1968. Lloydia, 33, suppl..3A.

12. SMOLENSKI, S.J., SILINIS, H. \& FARNSWORTH, N.R. -1974- Alkaloid screening. IV. Lloydia, 37:30.

13. SMOLENSKI, S.J., SILINIS, J. \& FARNSWORTH, N.R. -1972- Alkaloid screening. I. Llovdia, 35:1.

14. El-MASnY, S., El-GUAZOOLY, M.G., OMAR, A.A., KHAFAGY, S.M. \& PHILLIPSON, J.D. - 1981- Alkaloids from Egyptian Papaver rhoeas. Planta Med. 41:61.

15. CASTEDO, L., LOPEZ, S. \& VILLAVERDE, C. -1989- Alcaloides de Sarcocapnos baetica. Anal. Quim. 85C:48.

16. GOnZAlEZ, A.G., DE LA FUENTE, G., DIAZ, R., JONES, P.G. \& SHELDRICK, G.M. -1983- The structure of pentagydine. A new diterpenoid alkaloid. Tet. Letters, 24:959.

17. GARCIA, A.I. -1989- Evaluación, aislamiento y determinación estructural de alcaloides en la Flora de Andalucía. Tesis Doctoral. Universidad de Málaga.

18. CASTEDO, L. \& SUAU, R. -1986- The Cularine alkaloids, in The Alkaloids, Vol. 29. Ed. A. Brossi. Academic Press. Florida.

19. MATTOCKS, A.R. - 1986- Chemistry and toxicology of pyrrolizidine alkaloids. Academic Press. London.

20. KINGHORN, A.D. \& SMOLENSKY, A.D. -1981- Alkaloids of Papilionoideae in Advances in Legume systematics. Ed. R.M. Polhill \& P.H. Raven. Surrey.

21. PELLETIER, S.W. \& MODY, N.V. -1981- The Chemistry of C-20 diterpenoid alkaloids in The Alkaloids. Vol. 18. Ed. R.H.F. Manske \& R.G.A. Rodrigo. Academic Press. Florida.

22. SOUTHON, I.W. \& BUCKINGHAM. J. -1989- Dictionary of Alkaloids. Chapman \& Hall. London.

23. SLAVIK, J. -1963- Alkaloide der Mohngewächse (Papaveraceae). Ueber die alkaloidie aus Papaver dubium und über die Konstitution des aporheines. Collec. Czech. Chem. Commun, 28:1738.

24. MONTGOMERY, C.T., BRUCE, K.C. \& SHAMMA, M. -1983- The rhoeadine alkaloids. Lloydia, 46:441.

(Aceptado para su publicación el 15 de marzo de 1990)

Dirección de los autores: R. SUAU, A.I. GARCIA \& R. RICO: Dpto. de Química Orgánica. Facultad de Ciencias. Universidad de Málaga. B. CABEZUDO, J.M. NIETO \& A.E. SALVO: Dpto. Biología Vegetal. Facultad de Ciencias. Universidad de Málaga. 


\begin{tabular}{|c|c|c|c|c|c|c|}
\hline \multirow[t]{3}{*}{ FAMILIA-ESPECIE } & \multirow{3}{*}{\begin{tabular}{|c|} 
PARTE \\
DE LA \\
PLANTA
\end{tabular}} & \multicolumn{3}{|c|}{ ALCALOIDES } & \multirow{3}{*}{ CONCLUSION } & \multirow{3}{*}{\begin{tabular}{|c} 
ESTUDIOS \\
PREVIOS
\end{tabular}} \\
\hline & & \multicolumn{2}{|c|}{ TERCIARIOS } & \multirow{2}{*}{\begin{tabular}{|c|} 
CUATERNARIOS \\
ENSAYOS \\
CUALITATIVOS \\
\end{tabular}} & & \\
\hline & & \begin{tabular}{|c|} 
ENSAYOS \\
CUALITATIVOS \\
\end{tabular} & \begin{tabular}{|c|} 
VALORACION \\
CUANTITATIVA \\
\end{tabular} & & & \\
\hline ABIETACEAE & & & & & & \\
\hline $\begin{array}{l}\text { Abies pinsapo } \\
\text { AMARYLLIDACEAE }\end{array}$ & PA & + & 0,7 & $(+)$ & $\mathrm{P}$ & 4 \\
\hline Narcissus papyraceus & & & & & & \\
\hline subsp. polyanthus & PT & +++ & 2,9 & + & $\mathrm{P}$ & \\
\hline & B & +++ & 2,0 & + & $\mathrm{P}$ & \\
\hline APIACEAE & PA & +++ & 5,4 & + & $\mathrm{P}$ & 5 \\
\hline Bupleurum fruticescens & PA & $(+)$ & & - & $\mathrm{N}$ & \\
\hline Feula communis & PA & + & 0,2 & - & $\mathrm{D}$ & \\
\hline APOCINACEAE & & & & & & \\
\hline Vinca difformis & PA & +++ & 2,5 & + & $\mathrm{P}$ & 6 \\
\hline ASTERACEAE & & - & & - & $\mathrm{N}$ & \\
\hline $\begin{array}{l}\text { Inula crithmoides } \\
\text { Launea resedifolia }\end{array}$ & $\begin{array}{l}\mathrm{PT} \\
\mathrm{PA}\end{array}$ & 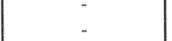 & & . & $\mathrm{N}$ & \\
\hline $\begin{array}{l}\text { Launea resedifolia } \\
\text { Santolina chamaecyparissus }\end{array}$ & $\mathrm{PA}$ & + & 0,3 & - & D & 6 \\
\hline $\begin{array}{c}\text { Santolina chamaecyparissus } \\
\text { BRASSICACEAE }\end{array}$ & & & & & & \\
\hline Moricandia arvensis & PT & + & 0,1 & + & D & \\
\hline Moricandia moricandioides & PT & - & & - & N & \\
\hline CARYOPHYLLACEAE & & & & & & \\
\hline Arenaria delaguardiae & PA & $(+)$ & & - & $\mathrm{N}$ & \\
\hline Dianthus broteri & PA & + & 0,1 & + & D & \\
\hline Silene colorata $\quad$ var. baetica & PT & - & & - & $\mathrm{N}$ & \\
\hline CELASTRACEAE & & & & & & \\
\hline Maytenus senegalensis (a) & PA & - & & - & $\mathrm{N}$ & 7 \\
\hline Maytenus senegalensis (b) & PA & - & & - & $\mathrm{N}$ & 7 \\
\hline EPHEDRACEAE & & & & & & \\
\hline Ephedra fragilis & PA & - & & - & $\mathrm{N}$ & \\
\hline FABACEAE & & & & & & \\
\hline Anthyllis cytisoides & PA - & & - & $\mathrm{N}$ & 8 & \\
\hline Anthyllis gerardii & $\mathrm{PA}$ & - & & - & $\mathrm{N}$ & 8 \\
\hline Anthyllis podocephala & PA & $(+)$ & & - & $\mathrm{N}$ & \\
\hline Astragalus lisitanicus & PA & $(+)$ & & - & $\mathrm{N}$ & \\
\hline Coronilla juncea & PA & - & & - & $\mathrm{N}$ & 8 \\
\hline Genista linifolia & PA & +++ & 4,7 & + & $\mathrm{P}$ & 8 \\
\hline Genista spartioides & PA & t++ & 0,4 & +++ & $P$ & \\
\hline Hedysarum coronarium & PA & ++ & 1,3 & + & P & \\
\hline Hedysarum humile & $\mathrm{PA}$ & $(+)$ & & - & $\mathrm{N}$ & 8 \\
\hline Hippodrepis rupestre & PA & + & 0,2 & - & D & \\
\hline Hippodrepis scabra & PA & + & 0,1 & $(+)$ & D & \\
\hline Lupinus micranthus & $\mathrm{PA}, \mathrm{F}$ & +++ & 5,0 & + & $P$ & 4 \\
\hline FAGACEAE & & & & & $N$ & 8 \\
\hline $\begin{array}{l}\text { Quercus faginea } \\
\text { LAMIACEAE }\end{array}$ & PA & - & & - & $\mathrm{N}$ & 8 \\
\hline $\begin{array}{l}\text { LAMIACEAE } \\
\text { Calamintha silvatica }\end{array}$ & & & & & & . \\
\hline $\begin{array}{l}\text { Calamintha silvatica } \\
\text { subsp. ascendens }\end{array}$ & PI & $(+)$ & & - & $\mathrm{N}$ & \\
\hline Cleonia lusitanica & PA & - & & - & $N$ & \\
\hline Lavandula stoechas & PA & $(+)$ & & $(+)$ & $\mathrm{N}$ & 9 \\
\hline Salvia barrelieri & PI & - & & - & $\mathrm{N}$ & \\
\hline Salvia verbenaca & $\mathrm{PA}$ & - & & - & $\mathrm{N}$ & 10 \\
\hline Satureja salzmannii & PA & $(+)$ & & - & $\mathrm{N}$ & \\
\hline Sideritis arborescens & & & & & & \\
\hline subsp. arborescens & $\mathrm{PA}$ & + & 0,2 & $(+)$ & D & \\
\hline Sideritis incana & & & & & & \\
\hline subsp. virgata (a) & PT & - & & - & $\mathrm{N}$ & \\
\hline $\begin{array}{l}\text { Sideritis incana } \\
\text { subsp. virgata (b) }\end{array}$ & $\dot{\mathrm{P}} \mathbf{I}$ & - & & - & $\mathrm{N}$ & \\
\hline Thymus zygis & & & & & & \\
\hline subsp. gracilis & PA & $(+)$ & & - & $\mathrm{N}$ & \\
\hline
\end{tabular}

Tabla I. 


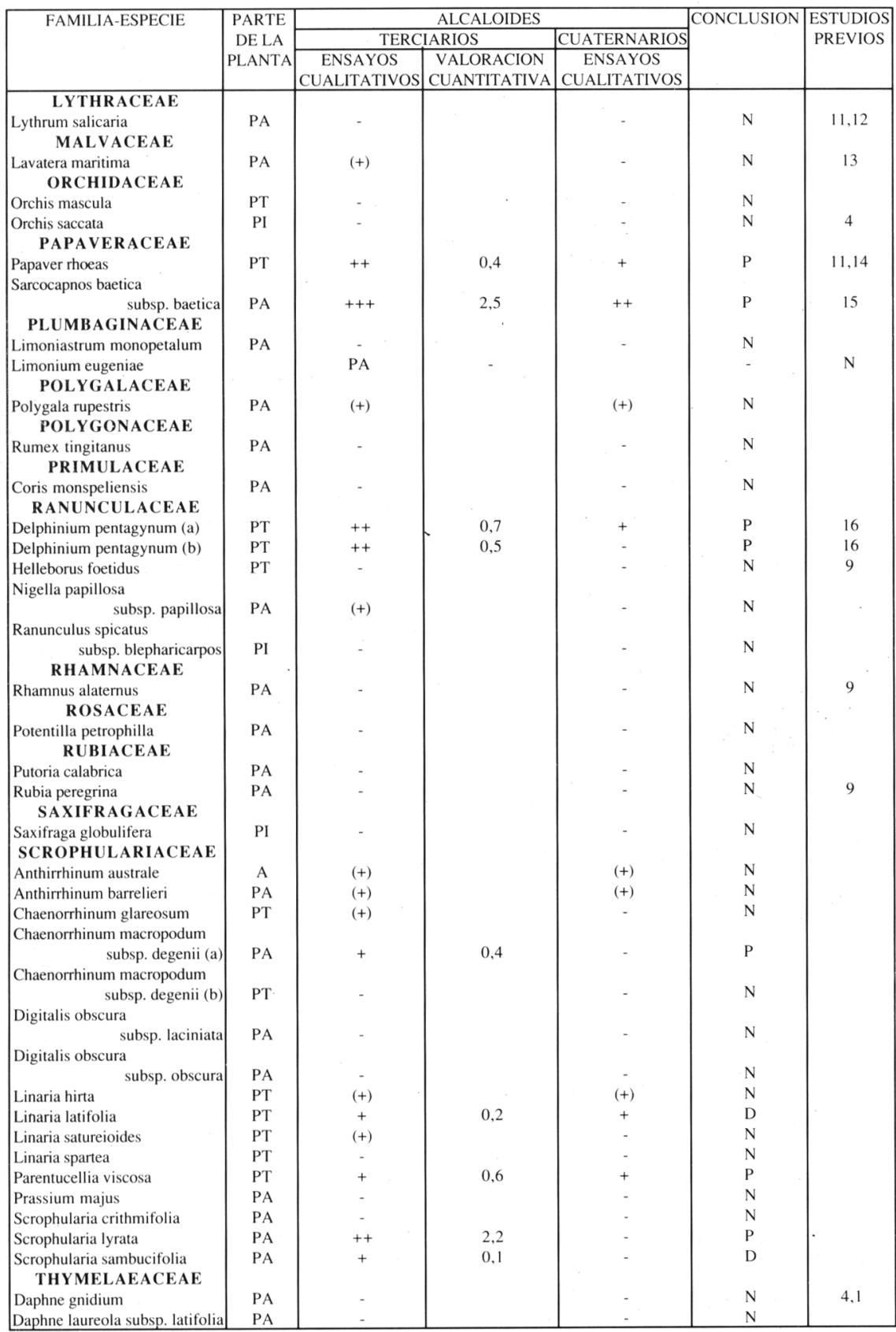

Tabla I (Continuación). 


\begin{tabular}{|c|c|c|}
\hline ESPECIE & \begin{tabular}{|c|}
$\mathrm{m} / \mathbf{z}(\%)$ \\
\end{tabular} & REFERENCIAS \\
\hline Abies pinsapo & $\begin{array}{l}295(6), 285(6), 253(30), 239(8), 159(14), \\
121(40), 95(60), 55(100) .\end{array}$ & \\
\hline Centranthus macrosiphon & $\begin{array}{l}397(2), 396(3), 331(7), 313(5), 248(30), \\
203(15), 169(60), 95(100) .\end{array}$ & 2 \\
\hline $\begin{array}{l}\text { Chaenorrhinum macropodum } \\
\text { subsp. degenii (a) }\end{array}$ & $\begin{array}{l}328(18), 314(25), 252(24), 206(25), 170(37), \\
117(100) .\end{array}$ & \\
\hline Chaenorrhinum villosum & $\begin{array}{l}407(5), 337(3), 307(2), 279(10), 262(3), \\
207(4), 167(35), 149(100) .\end{array}$ & 1 \\
\hline Delphinium gracile & $\begin{array}{l}476(3), 418(15), 358(10), 326(15), 280(6) \\
210(15), 180(25), 167(30), 105(100) .\end{array}$ & 2 \\
\hline Delphinium pentagynum (a) & $\begin{array}{l}436(4), 420(3), 326(3), 222(4), 216(10) \\
177(27), 149(100)\end{array}$ & \\
\hline Hedysarum coronarium & $\begin{array}{l}\text { 298(18), 286(12), 272(38), 270(62), 255(13), } \\
194(10), 181(14), 180(14), 150(100), 137(60) .\end{array}$ & \\
\hline Mandragora autumnalis & $\begin{array}{l}405(6), 345(10), 325(20), 281(16), 192(60) \\
177(60), 138(70), 125(100)\end{array}$ & 2 \\
\hline Narcissus cantabricus & $\begin{array}{l}357(3), 345(2), 331(18), 316(18), 315(10) \\
314(35), 301(22), 298(11), 281(3), 271(35) \\
247(45), 240(60), 238(100), 181(30), 115(32)\end{array}$ & 2 \\
\hline $\begin{array}{l}\text { Narcissus papyraceus } \\
\text { subsp. polyanthus }\end{array}$ & $\begin{array}{l}331(17), 317(50), 301(10), 276(18), 262(100) \\
247(60), 238(33), 226(50), 128(30)\end{array}$ & \\
\hline Papaver rhoeas & $\begin{array}{l}383(16), 369(12), 368(19), 335(16), 206(40) \\
190(80), 177(100), 163(60), 148(60)\end{array}$ & \\
\hline Papaver suaveolens & $\begin{array}{l}369(2), 354(3), 347(2), 339(3), 338(4), 333(10), \\
332(17), 287(11), 206(17), 192(10), 190(15), \\
178(20), 164(100) .\end{array}$ & 1 \\
\hline Parentucelia viscosa & $\begin{array}{l}329(2), 314(2), 299(4), 204(4), 177(4), 137(10) \\
122(40), 105(100) .\end{array}$ & \\
\hline $\begin{array}{l}\text { Platicapnos tenuiloba } \\
\text { subsp. tenuiloba }\end{array}$ & $\begin{array}{l}355(80), 354(100), 340(65), 339(50), 338(70), \\
324(56), 308(32), 281(33), 265(26) .\end{array}$ & 2 \\
\hline Santolina canescens (a) & $\begin{array}{l}334(5), 246(4), 230(8), 215(6), 163(17) \\
135(26), 95(100)\end{array}$ & 1 \\
\hline $\begin{array}{l}\text { Sarcocapnos baetica } \\
\text { subsp. baetica }\end{array}$ & $\begin{array}{l}355(4), 354(6), 353(8), 333(10), 332(16), \\
327(38), 325(10), 312(18), 310(18), 296(12), \\
\text { 294(12), 281(18), 192(30), 190(20), 177(20), } \\
\text { 163(28), 148(100). }\end{array}$ & \\
\hline $\begin{array}{l}\text { Senecio pyrenaicus } \\
\text { subsp. granatensis }\end{array}$ & $\begin{array}{l}353(3), 337(6), 266(5), 211(35), 140(80), \\
96(35), 82(100) .\end{array}$ & 1 \\
\hline Scrophularia lyrata & $\begin{array}{l}391(10), 300(2), 231(5), 189(8), 178(40), \\
161(100) .\end{array}$ & \\
\hline Viola crassiuscula & $\begin{array}{l}\text { 418(5), 281(2), 277(7), 243(20), 224(15), } \\
210(14), 197(20), 167(35), 84(100) .\end{array}$ & 2 \\
\hline
\end{tabular}

\section{Tabla II.}

